\title{
Macroprolactinoma appearing after resolution of hydrocephalus
}

\author{
Oscar D Bruno1,2, Ricardo Fernández Pisani³, Gabriel Isaac and Armando Basso³ \\ 1Division of Endocrinology, ${ }^{2}$ Fundacion de Endocrinologia, and ${ }^{3}$ Division of Neurosurgery, \\ Hospital de Clínicas, Universidad de Buenos Aires, Buenos Aires, Argentina
}

Correspondence should be addressed to O D Bruno

Email

bodomingo@intramed.net

\section{Summary}

The role of mechanical forces influencing the growth of a pituitary adenoma is poorly understood. In this paper we report the case of a young man with hyperprolactinaemia and an empty sella secondary to hydrocephalia, who developed a macroprolactinoma following the relief of high intraventricular pressure.

\section{Learning points:}

- The volume of a pituitary tumour may be influenced not only by molecular but also by local mechanical factors.

- Intratumoural pressure, resistance of the sellar diaphragm and intracranial liquid pressure may play a role in the final size of a pituitary adenoma.

- The presence of hydrocephalus may hide a pituitary macroadenoma.

\section{Background}

Factors controlling pituitary adenoma growth and aggressiveness are still poorly understood. The significance of molecular alterations acting through changes in the regulatory mechanisms of the cell cycle and subsequently in the growth pattern of pituitary adenomas is currently being investigated (1). However, to the best of our knowledge, the role of physical forces as conditioning factors in tumour growth has not yet been considered. In this study, we report a clinical case suggesting the possibility of such a role in a patient with a pituitary prolactinoma.

\section{Case presentation}

A 24-year-old man first consulted because of a progressive loss of libido and erectile dysfunction that had started at the age of 18. He had also had slow mentation and illdefined psychomotor troubles since the age of 15 . Physical examination was normal with the exception of scarce facial beard which he shaved sporadically; his weight was $79 \mathrm{~kg}$, height, $172 \mathrm{~cm}$ and blood pressure, 120/70 $\mathrm{mmHg}$.
The ophthalmologic evaluation showed normal visual acuity (10/10 in both eyes) but a bilateral papilledema.

\section{Investigations}

Adrenal and thyroid function tests were within the range of normalcy. Testosterone level was $3.4 \mathrm{ng} / \mathrm{mL}$ (normal value (nv): 3.5-8.0). The main finding was a serum prolactin (PRL) concentration of 880 and $1000 \mathrm{ng} / \mathrm{mL}$, on two separate occasions suggesting the diagnosis of a prolactinoma. However, instead of a pituitary adenoma, MRI revealed a very important ventricular dilatation and a partially empty sella with herniation of the suprasellar cisternae, the third ventricle and the optic chiasm into the pituitary fossae; idiopathic aqueductal stenosis was diagnosed (Fig. 1).

The patient was hospitalized and 24-h monitoring of intracranial pressure (IP) showed a mean figure of $16 \mathrm{mmHg}$ (normal values $<13 \mathrm{mmHg}$ ) (2). His clinical condition deteriorated with the loss of communication, disorientation and prostration. 


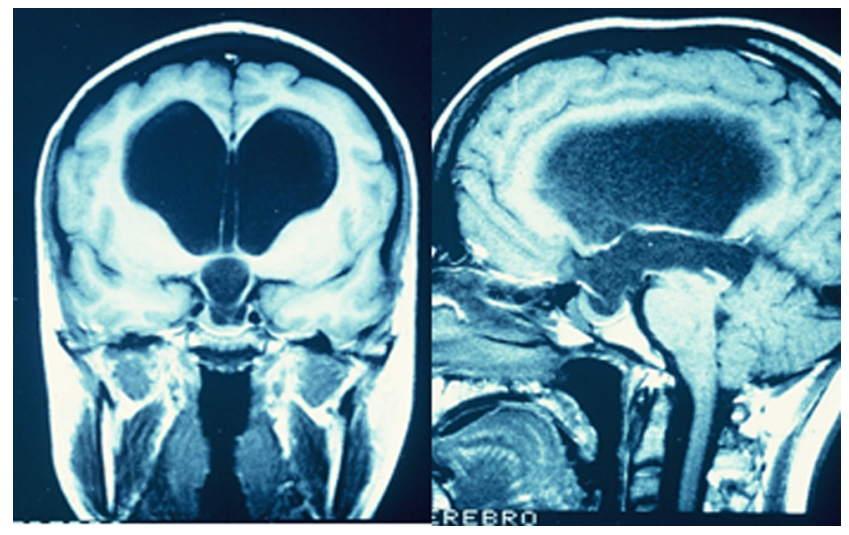

Figure 1

Magnetic resonance imaging (coronal and saggital views) of patient MC showing a huge dilation of the ventricular system and sellar arachnoidocele (see text for details).

\section{Treatment}

After two unsuccessful attempts to place shunting devices (a medium pressure valve and a flow-dependent valve respectively), a ventricular-peritoneal shunt using a mean-pressure valve was performed. A progressive improvement began on the sixth postoperative day with recovery of oral language, and continued until the total normalization of all neuropsychological functions. At that time, IP was $7 \mathrm{mmHg}$, MRI showed a ventricular dilation reduction and a $13 \mathrm{~mm}$ image compatible with pituitary macroadenoma was now evident (Fig. 2); serum PRL was $953 \mathrm{ng} / \mathrm{mL}$. After three months, an additional reduction of the ventriculomegaly was observed (Fig. 3) but serum PRL remained high at $400-500 \mathrm{ng} / \mathrm{mL}$, despite treatment with increasing doses of bromocryptine (up to $25 \mathrm{mg}$ /day).

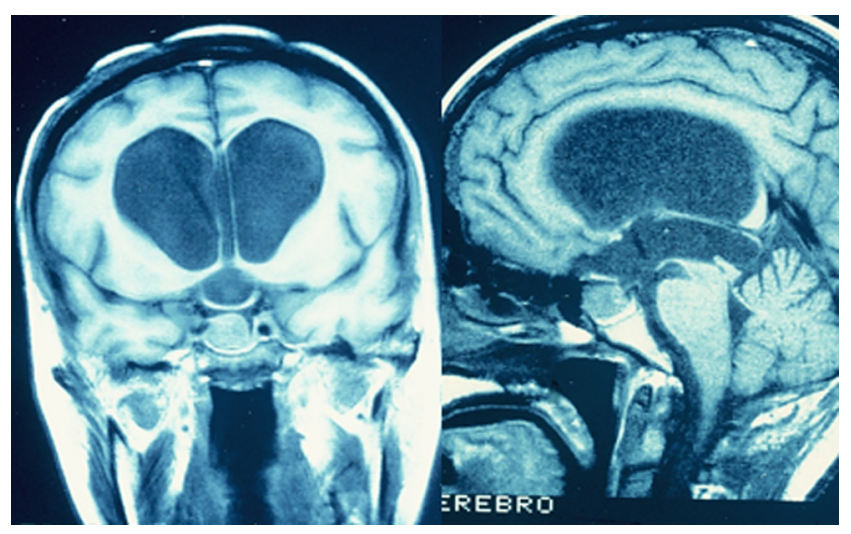

\section{Figure 2}

Magnetic resonance imaging (coronal and sagittal views) of patient MC showing slight reduction of ventricular dilation and appearance of image of pituitary macroadenoma one week after ventriculo-peritoneal shunt (see text for details).

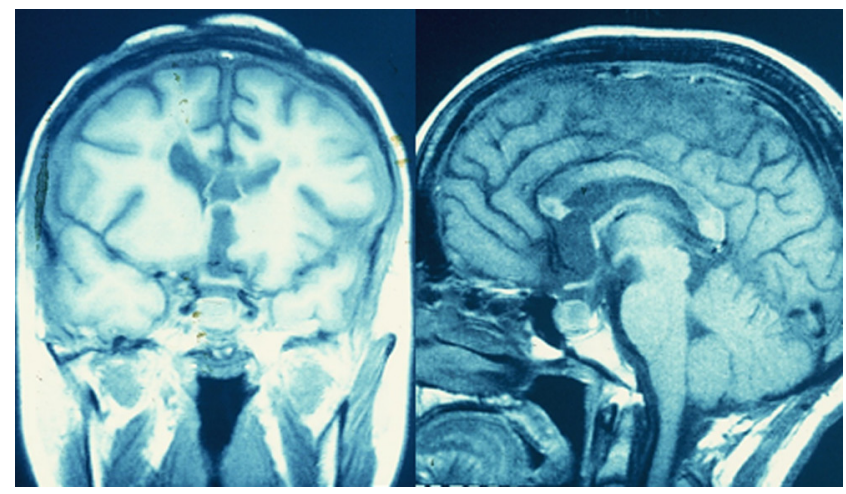

Figure 3

Magnetic resonance imaging (coronal and sagittal views) of patient MC showing normalization of the ventricular system but persistence of the pituitary adenoma after three months of placement of ventriculoperitoneal derivation (see text for details).

This treatment had to be discontinued because of the development of psychotic signs which subsided after a few days. A trial with cabergoline was indicated and then the patient was operated on by the transsphenoidal approach to resect a prolactinoma confirmed by the pathologist with immunomarcation showing strong positivity for PRL.

\section{Outcome and follow-up}

In the following months, serum PRL fell to between 90 and $200 \mathrm{ng} / \mathrm{mL}$. After discharge, he was treated for panhypopituitarism with levothyroxine $100 \mu \mathrm{g} / \mathrm{day}$, hydrocortisone $20 \mathrm{mg} /$ day, testosterone gel 1 application/ day. He was lost to monitoring for a very long period but reappeared at the beginning of 2016, almost 20 years after his first consultation. He was clinically stable under treatment and a physical exam conducted on him was normal. MRI showed no pituitary tumour but a moderate dilation of
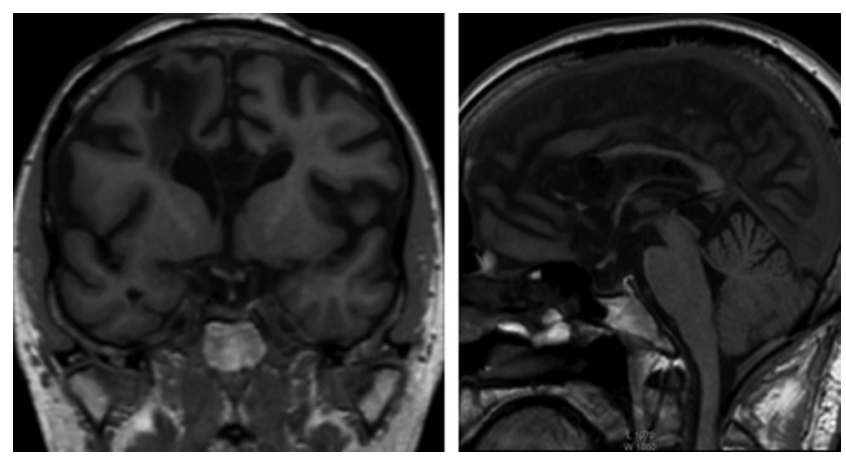

Figure 4

Magnetic resonance imaging (coronal and sagittal views) of patient MC obtained 20 years after pituitary surgery showing a moderate dilation of the ventricular system and absence of remnants of pituitary tumour (see text for details). 
the ventricular system (Fig. 4). Hormonal lab showed undetectable serum LH, FSH, GH and cortisol; prolactin level was $61 \mathrm{ng} / \mathrm{mL}$. The long-term patient's evolution has been satisfactory and he lives and works normally.

\section{Discussion}

Around $80 \%$ of prolactinomas are microadenomas, though macroadenomas are frequently observed in young male patients (3). Normal mean intrasellar pressure is not known but it is unlikely to exceed normal intracranial pressure (ICP). An ICP value below $13 \mathrm{mmHg}$ characterizes normal CSF circulation; above $18 \mathrm{mmHg}$ the CSF circulation is clearly disturbed; between $13 \mathrm{mmHg}$ and $18 \mathrm{mmHg}$ there is a grey zone (2). On the other hand, it is known that pituitary intratumoral pressure can be variable, depending on the size of the tumour. In a two-centre study, intrasellar pituitary tissue pressure (ISP) was measured during transsphenoidal surgery in 107 patients with pituitary adenomas or intrasellar cysts; 'normal' ISP in patients with microadenomas less than $5 \mathrm{~mm}$ diameter or a partial empty sella was $12 \pm 3 \mathrm{mmHg}$. Raised ISP of more than $15 \mathrm{mmHg}$ was found in $75 \%$ of the cases (4). On the other hand, little is known about the possible influence of variable resistance of the anatomical surrounding structures on the growth of pituitary tumours. The sellar diaphragm is composed of two layers of dura mater forming the upper limit of the sella turcica, but its resistance to pressure on both sides is difficult to define; it could be because anatomically weakened resistance is involved in the pathogenesis of primary empty sella (5). In the same way, individual variation in the resistance of the sellar diaphragm can partially explain the greater or smaller suprasellar expansion of pituitary adenomas. There is also a remarkable variation in the morphology of the diaphragm opening which could explain the growth of pituitary tumours towards the cavernous sinus or towards the suprasellar region (6).

Our patient presented a mean ICP of around $16 \mathrm{mmHg}$ accompanied by a clear clinical intracranial hypertensive syndrome and imaging of a huge hydrocephalus which prompted neurosurgical treatment. Interestingly, in the present case higher than normal IP could have impeded tumour expansion, without influencing PRL secretion. We assumed that the pituitary adenoma was not the cause of hydrocephalus because of its moderate size. Besides, the tumour expansion took place shortly after intracranial pressure decrease, even before normalization of the ventricular dilation, leading to the clear visualization of a typical macroadenoma image. The endocrine clinical findings in this case are not different from those shown in the usual cases of prolactinoma, but the main interest of the observation is the unusual form of presentation which allowed us to speculate on the role of mechanical factors influencing the size of a pituitary adenoma.

Declaration of interest

The authors declare that there is no conflict of interest that could be perceived as prejudicing the impartiality of the research reported.

\section{Funding}

This research did not receive any specific grants from any funding agencies in the public, commercial or not-for-profit sectors.

\section{Patient consent}

Written informed consent has been obtained from the patient for the publication of the case report.

\section{Author contribution statement}

$\mathrm{Dr} O \mathrm{D}$ Bruno is a consultant endocrinologist and professor of medicine who was in charge of the patient together with $\mathrm{Dr} \mathrm{G}$ Isaac. Dr Fernandez Pisani and Prof. A Basso were the neurosurgeons responsible for the surgical procedures. All the authors reviewed and revised the present manuscript.

\section{References}

1 Monsalves E, Larjani S, Loyola Godoy B, Juraschka K, Carvalho F, Kucharczyk W, Kulkarni A, Mete O, Gentili F, Ezzat S, et al. 2014 Growth patterns of pituitary adenomas and histopathological correlates. Journal of Endocrinology and Metabolism 99 1330-1338. (doi:10.1210/jc.2013-3054)

2 Czosnyka M \& Pickard JD 2004 Monitoring and interpretation of intracranial pressure. Journal of Neurology, Neurosurgery, and Psychiatry 75 813-821. (doi:10.1136/jnnp.2003.033126)

3 Gillam MP, Molitch ME, Lombardi G \& Colao A 2006 Advances in the treatment of prolactinomas. Endocrine Reviews 27 485-534. (doi:10.1210/er.2005-9998)

4 Lees PD, Fahlbusch R, Zrinzo A \& Pickard JD 1994 Intrasellar pituitary tissue pressure, tumour size and endocrine status - an international comparison in 107 patients. British Journal of Neurosurgery 8 313-318. (doi:10.3109/02688699409029619)

5 Saindane AM, Lim PP, Aiken A, Chen Z \& Hudgins PA 2013 Factors determining the clinical significance of an 'empty' sella turcica. American Journal of Roentgenology 200 1125-1131. (doi:10.2214/ ajr.12.9013)

6 Campero A, Martins C, Yasuda A \& Rothon AL Jr 2008 Microsurgical anatomy of the diaphragma sellae and its role in directing the pattern of growth of pituitary adenomas. Neurosurgery 62 717-723. (doi:10.1227/01.neu.0000317321.79106.37) 